\title{
Glycogen storage disease type iv: a case with histologic findings in placental tissue from first trimester miscarriage
}

\author{
Anastasia Papakonstantinou, Konstantinos Zacharis, Stavros Kravvaritis, \\ Theodoros Charitos, Eleni Chrysafopoulou, Anastasia Fouka
}

Department of Obstetrics and Gynecology, General Hospital of Lamia, Lamia, Greece

Correspondence

Correspondence should be addressed to Konstantinos Zacharis; zaxarisk@yahoo.com

\section{Abstract}

Glycogen storage disease Type IV is a rare hereditary autosomal recessive disorder caused by deficient enzymatic activity of glycogen branching enzyme (GBE) which is encoded by GBE1 gene. We hereby report the case of a 32-year-old woman presented with a first-trimester miscarriage. The histologic findings of the placental tissue included intracytoplasmic inclusion vacuoles suggested GSD-IV. The diagnosis was confirmed by the genetic analysis of the parents, in which mother was found to be carrier of a GBE1 mutation. This variable disorder can be diagnosed by histopathology of the placenta but for its confirmation and prevention in subsequent pregnancies, genetic analysis is needed.

Key words: Glycogen storage disease Type IV, Intracytoplasmic inclusion vacuoles, placenta, first trimester, early miscarriage

\section{Introduction}

Glycogen storage disease Type IV (GSD-IV) or Andersen disease is a rare autosomal recessive metabolic disorder caused by glycogen branching enzyme (GBE) deficiency leading to the accumulation of insoluble abnormal glycogen with fewer branching points (polyglucosan-like) in the affected tissue. GBE is encoded by the glycogen branching enzyme 1 (GBE 1) gene located on chromosome 3p14. GBE 1 mutations are the only known to cause GSD-IV ${ }^{1-2}$. The clinical presentation of the disease and the tissue involvement are heterogenous. The GSD-IV phenotype ranges from mild to severe, even fatal ${ }^{1}$. Placental involvement was initially described in 2008 in two cases with fetuses delivered during the third trimester of pregnancy ${ }^{3}$. Early miscarriages due to placental polyglucosan-bodies accumulation have also been described ${ }^{4}$. In this case report, we present placental histopathology and genetic data of another GSD-IV related first trimester miscarriage. 


\section{Case report}

A 32-year-old healthy woman presented to the obstetric outpatient department for routine antenatal care scan. Ultrasound examination revealed no cardiac heartbeat at gestational week 9+ $1 \mathrm{~d}$. A medical induced miscarriage occurred, followed by curettage and tissue was histologically examined.

\section{Placental Histopathology}

The histologic findings confirmed the presence of a first-trimester abortion. Embryonic structures were not recognized on the aspirated material. Microscopic examination of the placental tissues demonstrated irregular trophoblastic layer and clusters of signet-ring cells in the intervillous space surrounded by trophoblastic cells or adjacent to chorionic villi (Figure 1). These signet ring like cells contained Intracytoplasmic inclusion vacuoles with light eosinophilic reaction in Hematoxylin-Eosin staining. Inclusion bodies were also strongly reactive to Periodic acid-Schiff (PAS) and PAS diastase staining, while negatively reactive in alcian blue staining. These findings were compatible with polyglucosanlike deposits in placental tissue, raising suspicion of GSD-IV. Typical inclusion bodies were not identified by electron microscopy since the affected tro-

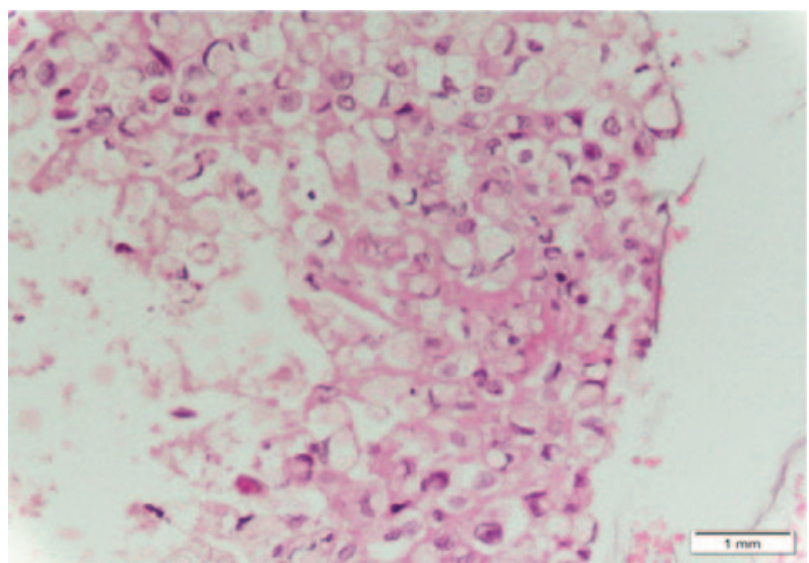

phoblastic cells were sparsely scattered within placental tissue.

\section{Molecular Genetic Analysis}

A constitutional genetic analysis was performed on both parents after genetic counseling and informed consent. Genetic testing was performed using polymerase chain reaction (PCR) and next generation sequencing (NGS) methods. Mother was found to be a heterozygote for a novel GBE1 mutation p.Pro159Leu (c.476 C>T), which results in a replacement of proline (Pro) amino acid by leucine (Leu) in position 159. This GBE1 mutation causes GSD-IV has not been previously described. DNA analysis of the parental specimen did not reveal any GBE1 mutation.

\section{Discussion}

GSD-IV is a rare disease, representing 3\% of all glycogen storage disease and may be under-diagnosed $^{5}$. Rarely, as in our case, GSD-IV may be caused by a heterozygous GBE1 mutation and a heterozygous deletion of one or more exons of the allele GBE1 gene $^{6-9}$. GSD-IV may affect early pregnancies leading to trophoblastic damage and early fetal loss ${ }^{5}$. Placental involvement underlines the importance of

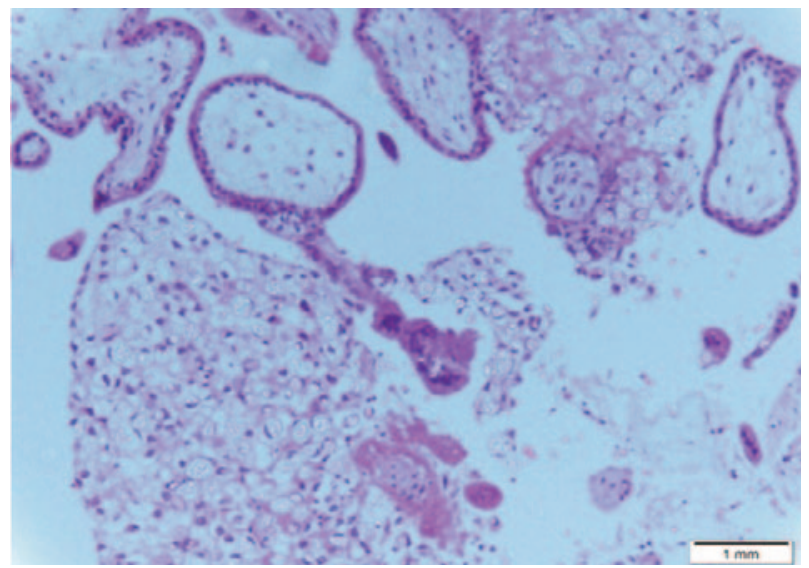

Figure 1. Trophoblastic cells with Intracytoplasmic vacuoles (signet-ring cells). 
histopathologic examination of the placenta in cases with early miscarriages. When vacuolated cells are discovered during histopathology of the placental tissue, GSD-IV should be suspected ${ }^{4}$. However, genetic analysis of both miscarriage material and parents is required to confirm the diagnosis. Prenatal genetic testing should be performed in subsequent pregnancies $^{5}$.

\section{Consent}

Informed consent was obtained.

\section{Conflicts}

The authors declare no conflicts of interest.

\section{Authors' contribution}

$\mathrm{AP}, \mathrm{KZ}$ and EC wrote the first draft. SK, TC and AF critically reviewed and amended the draft. All authors approved of the final draft.

\section{References}

1. Moses SW, Parvari R. The variable presentations of glycogen storage disease type IV: a review of clinical, enzymatic and molecular studies. Curr Mol Med. 2002;2:177-88.

2. Akman HO, Karadimas C, Gyftodimou Y, Grigoriadou M, Kokotas $\mathrm{H}$, et al. Prenatal diagnosis of glycogen storage disease type IV. Prenat Diagn. 2006 Oct;26(10):951-5.

3. Konstantinidou AE, Anninos H, Dertinger S, Nonni A, Petersen M, et al. Placental involvement in glycogen storage disease type IV. Placenta. 2008 Apr;29(4):378-81.

4. Bendroth-Asmussen L, Aksglaede L, Gernow AB, Lund AM. Glycogen Storage Disease Type IV: A Case with Histopathologic Findings in FistTrimester Placental Tissue. Int J Gynecol Pathol.
2016 Jan;35(1):38-40.

5. Dainese L, Adam N, Boudjemaa S, Hadid K, Rosenblatt J, et al. Glycogen Storage Disease Type IV and Early Implantation Defect: Early Trophoblastic Involvement Associated with a New GBE1 Mutation. Pediatr Dev Pathol. 2016 Nov/Dec;19(6):512-515.

6. Raju GP, Li HC, Bali DS, Chen YT, Urion DK, et al. A case of congenital glycogen storage disease type IV with a novel GBE1 mutation. J Child Neurol. 2008 Mar;23(3):349-52.

7. Li SC, Hwu WL, Lin JL, Bali DS, Yang C, et al. Association of the congenital neuromuscular form of glycogen storage disease type IV with a large deletion and recurrent frameshift mutation. J Child Neurol. 2012 Feb;27(2):204-8.

8. Nolte KW, Janecke AR, Vorgerd M, Weis J, Schröder JM. Congenital type IV glycogenosis: the spectrum of pleomorphic polyglucosan bodies in muscle, nerve, and spinal cord with two novel mutations in the GBE1 gene. Acta Neuropathol. 2008 Nov;116(5):491-506.

9. Tay SK, Akman HO, Chung WK, Pike MG, Muntoni $F$, et al. Fatal infantile neuromuscular presentation of glycogen storage disease type IV. Neuromuscul Disord. 2004 Apr;14(4):253-60.
Received 1-6-2019

Revised 18-6-2019

Accepted 26-6-2019 